\title{
Estudio de Motivación de Colaboradores en las Medianas Empresas en la Ciudad de Xalapa, México
}

\section{Collaboration Motivation Study in Medium-sized Companies in the City of Xalapa, México}

Luis Alejandro Gazca-Herrera'; Ximena Montserrat Zárate-Hernández²

Alí Aminadab Mota-Hernández ${ }^{3}$

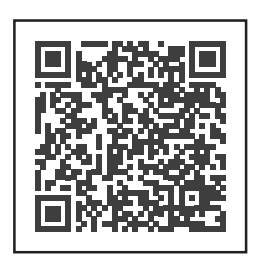

\section{Palabras claves:}

Colaboradores, Desmotivación, Maslow, Medianas empresas, Motivación, Necesidades.

Articulo de investigación:

Fecha de recepción:

26/03/2020

Fecha de aceptación: 04/06/2020

Esta publicación se encuentra bajo licencia:

Creative Commons ReconocimientoNoComercialSinObraDerivada 4.0 Internacional

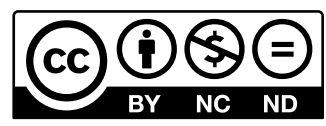

\section{Resumen}

La desmotivación en los colaboradores es una problematica que afecta el rendimiento laboral, presentado complicaciones en la salud emocional y psicológica; por lo que la productividad se ve mermada y las consecuencias se reflejan en elevados costos operativos. La presente investigación buscó identificar el grado de motivación que tienen los colaboradores en las medianas empresas de la ciudad de Xalapa, Veracruz, México, específicamente en las áreas funcionales de la estructura organizacional, se realizó el diagnóstico bajo el análisis de las teorías de motiviación y necesidades humanas de Maslow. Se procedió con una investigación de tipo no experimental, exploratoria, descriptiva y cuantitativa; se administró un instrumento de motivación debidamente validado con 20 ítems dividido por dimensiones que miden los elementos de la teoría de las necesidades y la motivación. Las unidades de análisis se determinaron con base en una población infinita que estuvo representada por

1 Licenciado en Sistemas Computacionales Administrativos, Maestro en Ciencias Administrativas y Doctor en Administración Pública, Universidad Veracruzana - Facultad de Contaduría y Administración, México, Igazca@ uv.mx, @Alex_Gazca, https://orcid.org/0000-0001-7637-2909

2 Licenciada en Sistemas Computacionales Administrativos, Universidad IUV - Universidad Virtual, México, ximena.zarate@iuv.edu.mx, @ximenazh, https://orcid.org/0000-0001-7042-0404

3 Estudiante de la Licenciatura en Sistemas Computacionales Administrativos, Universidad Veracruzana - Facultad de Contaduría y Administración, México, ahdez1195@gmail.com, https://orcid.org/0000-0003-3367-0388 


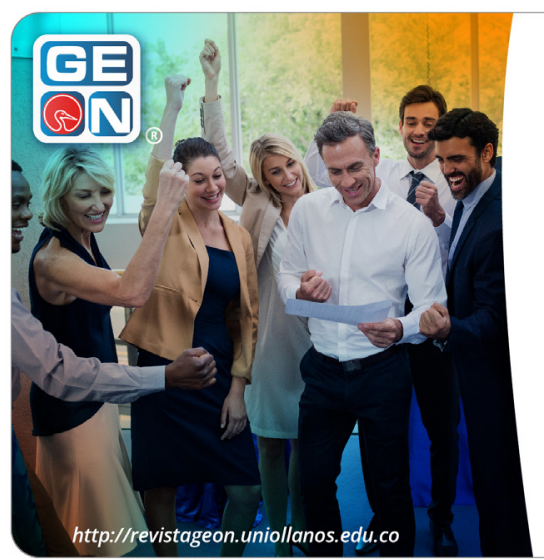

\section{$\stackrel{\text { Revista GEON }}{\mathbf{f} \text { in }} \mathbf{0} \mathbf{0}$}

66 más del $60 \%$ de los colaboradores no están motivados con su entorno laboral. 99

96 medianas empresas. Los resultados obtenidos arrojaron que el personal no siente que se cumplan en su mayoría sus necesidades de reconocimiento ni de autorrealización. La prueba de hipótesis estadística demostró que más del 60\% de los colaboradores no están motivados con su entorno laboral. Por lo ya expresado se concluyó que es necesario que se establezcan metas claras para poder aprovechar las potencialidades de los colaboradores, en el mismo sentido diseñar programas de incentivos, reconocimiento de logros personales y de ser necesario cambios en el planteamiento del trabajo.

Palabras claves: Colaboradores, Desmotivación, Maslow, Medianas empresas, Motivación, Necesidades.

\section{Códigos JEL: M12}

\section{Abstract}

The lack of motivation in employees is a problem that affects their work performance, having complications in their emotional and psychological health, regardless of the size or type of organization, productivity is reduced and the consequences are reflected in raising operating costs in general terms. This research sought to identify the degree of motivation that collaborators have in medium-sized companies in the city of Xalapa, Veracruz, Mexico, specifically in the functional areas of the organizational structure, aimed to make the diagnosis un- 
Cómo citar este artículo / Toreference this article:

Gazca Herrera, L., Zárate Hernández, X., \& Mota Hernández, A. (2020). Estudio de Motivación de Colaboradores en las Medianas Empresas en la Ciudad de Xalapa, México. Revista GEON (Gestión, Organizaciones Y Negocios), 7(2), 1-11. https://doi. org/10.22579/23463910.207 der the analysis of the theories of Motivation and human needs of Maslow to inquire in a general way how motivated they are and if they meet their needs according to this theory. A non-experimental, exploratory, descriptive and quantitative research was carried out; For this, a duly validated motivational instrument with 20 items divided by dimensions was administered, with indicators that measure the elements of the theory of needs and the motivation of the collaborators. The analysis units were determined based on an infinite population that was represented by 96 medium-sized companies, which were specifically surveyed in the functional area, being industrial, service and commercial companies. The results obtained showed that the surveyed personnel did not feel that their needs for recognition or self-fulfillment were mostly met, in the same sense, the statistical hypothesis test showed that more than $60 \%$ of the collaborators were not motivated by their work environment. Based on the aforementioned, it was concluded that it is necessary to establish clear goals both at the organizational and personal level in order to take advantage of the potential of the collaborators, in the same sense, design incentive programs, recognition of personal achievements and, if necessary, changes in the approach to work.

Keywords: Needs, Motivation, Maslow, Medium enterprises, Demotivation.

\section{Introducción}

La motivación es un elemento que las empresas deben fomentar, siendo esta, la que impulsa al individuo a realizar sus actividades bajo un contexto de satisfacción con su trabajo generando un mayor compromiso y sentido de pertenecía. Las razones por las que los trabajadores se encuentran motivados van evolucio- nando a lo largo del tiempo según vaya satisfaciendo desde sus necesidades más básicas hasta los aspectos de autorrealización.

La problemática que presentan las organizaciones es que no cuentan con planes de programas de desarrollo para los colaboradores, donde la participación de ellos en el progreso de la empresa es uno de los princi- 
pales pilares para las organizaciones, esta situación genera una desmotivación y falta de compromiso al interior de la organización.

Con base en la problemática descrita, el objetivo de esta investigación fue realizar una evaluación a las medianas empresas bajo el análisis de las teorías de motivación de las necesidades humanas de Maslow, y al igual que Rodríguez y Molero (2018) este estudio hasta el final en el sustento de esta aproximación investigativa obtendrá conclusiones tanto generales como particulares que podrán ser aplicadas a la práctica, cuando la organización diseñe programas de desarrollo organizacional.

\section{Contexto Teórico}

Según Robbins y Coulter (2005) la motivación se refiere a aquello que hace que la gente actúe o se comporte de determinadas maneras, pero también cuando se habla de motivación, no solo se hace referencia a las personas, sino también al sujeto forjador de dicha motivación. Por motivación se debe entender que es un proceso psicológico que se relaciona con el impulso (amplitud), dirección y persistencia de la conducta. La conducta motivada resulta de la identificación de dos grandes conjuntos de aspectos del trabajo que son motivadores de la conducta laboral: motivadores del entorno laboral y motivador del contenido del trabajo. Los primeros hacen referencia a las características del ambiente en donde tiene lugar la actividad laboral y son de naturaleza material y social (Arauz, Díaz, Gonzalez y Vasquez, 2018).
La motivación se integra por ciertos aspectos que determinan la actuación de los colaboradores en una organización, por ejemplo, para Martínez, Hernández, Torres-Flórez, García, y Montaño (2018) citando a Aguilera (2011) mencionan que el liderazgo participativo es uno de los factores que otorgan mayor motivación, satisfacción para obtener resultados positivos en el clima organizacional, en el mismo sentido Pineda (2020) de acuerdo con Gómez y Benito (2014) mencionan a otro elemento la comunicación interna, la cual es una herramienta para la motivación de los empleados y para transmitirles la información acerca de las actividades de la empresa, de sus logros y fracasos.

La Teoría de la Motivación Humana, propone una jerarquía de necesidades y factores que motivan a las personas; esta pirámide se define por cinco niveles considerando un orden jerárquico ascendente que puede determinar su capacidad de motivación y supervivencia (Quintero 2015).

Cada ser humano reacciona diferente, se desempeña de diferente manera dependiendo de cómo tenga satisfechas sus necesidades, de eso dependerá, como se desarrolle en sus actividades ya que según Maslow (1943) señala que las personas se encuentran motivadas por cinco tipos de necesidades: fisiológicas, de seguridad, sociales, autoestima y autorrealización.

Por lo tanto, conocer el impacto que tiene la teoría de las necesidades humanas de Maslow puede permitir sa- 
ber cómo las empresas motivan a sus colaboradores para llegar a tener todas sus necesidades satisfechas.

\section{Materiales y Método}

Se llevó a cabo una investigación aplicada, de campo, no experimental, exploratoria, explicativa y cuantitativa, con un enfoque, descriptivo ya que buscó caracterizar y describir aspectos propios de una determinada variable, al igual que Martínez (2015) el presente artículo se desarrolló basado en una investigación de tipo descriptiva, para lo cual se realizó la revisión conceptual y literaria pertinente en las principales revistas a nivel nacional e internacional sobre el concepto de motivación.

El diseño del instrumento se realizó bajo el criterio de la escala de Likert, considerando la forma eficaz para medir los indicadores de las dimensiones de motivación y la satisfacción de necesidades humanas, se creó un instrumento de veinte ítems que permitió obtener resultados sobre las dos dimensiones y los indicadores correspondientes. La fiabilidad y validez del instrumento se analizó con el coeficiente Alfa de Cronbach, el proceso de análisis se realizó mediante el software SPSS, este cálculo emplea el promedio de todas las correlaciones existentes entre los ítems del instrumento. El valor de alfa de Cronbach obtenido fue de .711 de acuerdo con la escala De Vellis citado por García y Cirilo (2006), esto representa un nivel respetable de confiabilidad.

Al no encontrar una fuente secundaria fidedigna de información (INEGI,
Secretaría de Economía, Bancomext, entre otros) para determinar la población (sujetos de estudio) de las medianas empresas en Xalapa, se definieron las unidades de análisis bajo una población infinita. Para la determinación del tamaño de la muestra se consideraron los siguientes dtos:

En donde:

n=tamaño de la muestra

e= Error de estimación máximo aceptado $=10 \%=.10$

$\mathrm{p}=$ Probabilidad de que ocurra el evento $=.5$

$q=(1-p)$ Probablidad de que no ocurra el evento

Z= Parámetro estadístico que depende de N (grado de precisión) = 95\% en tablas 1.96

$$
n=\frac{1.96^{2} * .5 * .5}{.1^{2}}
$$

El resultado de la muestra son 96 encuestas que se aplicaron a los colaboradores de las áreas funcionales de las medianas empresas de Xalapa.

\section{Resultados}

De las encuestas aplicadas 47\% fueron contestadas por hombres y $53 \%$ por mujeres; de los colaboradores 16\% tienen una edad entre 23 a 30 años, 44\% entre 31 a 45 años, 27\% tienen de 46 a 55 años y 14\% de 56 años en adelante.

En la gráfica 1, se puede observar que 62\% respondió que la empresa 
sí toma en cuenta sus necesidades y $38 \%$ que no.

Gráfica 1. La empresa toma en cuenta las necesidades de los colaboradores.

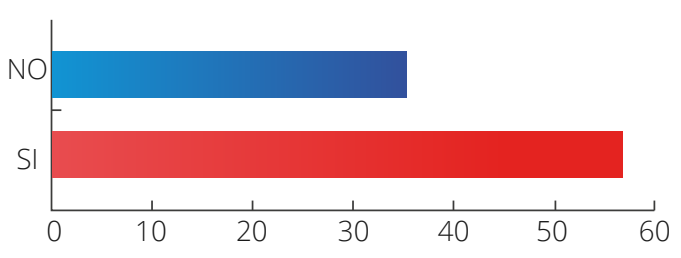

Fuente: Elaboración propia (2019)

En la gráfica 2, se puede visualizar que $74 \%$ de los colaboradores se encuentran muy satisfecho en su ámbito laboral, 19\% satisfecho y $7 \%$ insatisfecho.

Gráfica 2 ¿Cuál es su grado de satisfacción en su ámbito laboral?

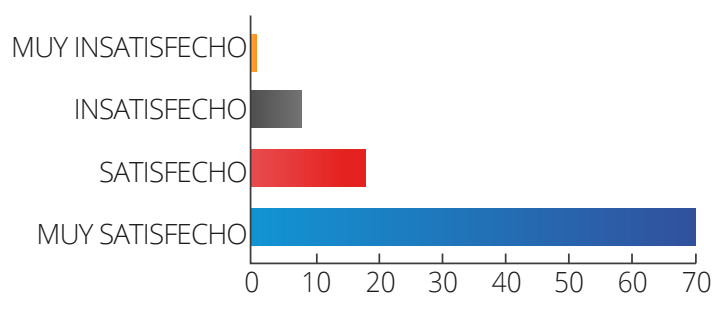

Fuente: Elaboración propia (2019)

Conforme a la teoría de Maslow referente a las necesidades fisiológicas (Necesidades básicas de la vida, aire, comida, bebida, refugio, calor, sexo, sueño, entre otros), de los colaboradores encuestados casi 6 de cada 10 considera que sus necesidades fisiológicas se cumplen en una percepción que supera el $75 \%$ de satisfacción.

Referente a la necesidad de seguridad (Seguridad física, de empleo, de recursos, moral, familiar, de salud, de propiedad privada, entre otros) de los colaboradores encuestados 5 de cada 10 considera que sus necesidades de seguridad se cumplen en un Orango superior al $75 \%$ de satisfacción.

En cuanto a las necesidades de afiliación o pertenencia (Amistad, afecto, pareja, raíces, relaciones sociales, pertenencia algún colectivo, entre otros) de los colaboradores encuestados 4 de cada 10 considera que sus necesidades de afiliación se cumplen en un rango superior al $75 \%$.

En las necesidades de reconocimiento (Autorreconocimiento, confianza, respeto, prestigio, logros, entre otros) de los colaboradores encuestados 3 de cada diez considera que sus necesidades de reconocimiento se cumplen en un rango superior al $75 \%$.

En lo referente a las necesidades de autorrealización (Crecimiento general, creatividad, visión global, espontaneidad, liderazgo, entre otros), de los colaboradores encuestados casi 3 de cada 10 considera que sus necesidades de autorrealización se cumplen en un rango superior al $75 \%$.

En la gráfica 3 sobre la tendencia de satisfacción de las necesidades, se puede apreciar, que en los porcentajes de satisfacción a menor nivel de la pirámide de necesidades los porcentajes son mayores, sin embargo conforme se va subiendo en los niveles de satisfacción los porcentajes van disminuyendo, sin duda esto debido a que con base en los resultados las 
necesidades de satisfacción en los niveles inferiores se pueden cubrir de manera más fácil, pero conforme se va subiendo es más difícil satisfacerlos, hay que recordar que a los colaboradores con quienes se aplicó el instrumento son de las áreas funcionales los que están en la parte media de una estructura organizacional.

Gráfica 3. Tendencia en la Satisfacción de las Necesidades

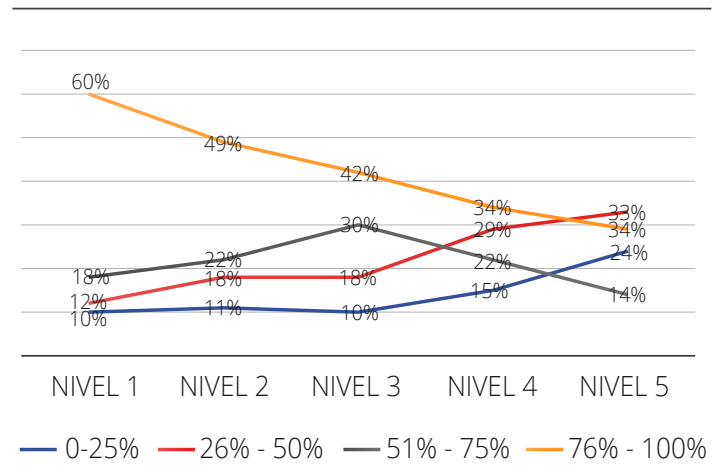

Fuente: Elaboración propia (2019)

Con base en los resultados y coincidiendo con Logroño y Cercas (2018) el conocer cuál es la motivación de un colaborador permite desarrollar estrategias a la hora de proponer planes de programas de desarrollo para los colaboradores, generando aspiraciones de superación manteniendo su motivación a largo plazo.

\section{Prueba de hipótesis estadística}

Las pruebas de hipótesis involucran diversos conceptos y términos que son necesarios comprender para su correcta aplicación e interpretación, las hipótesis estadísticas son afirmaciones acerca de los parámetros, por lo que para probar la validez de una hipótesis de investigación es necesario plantearla en término de hipótesis estadísticas. Por su parte, en el enfoque de Neyman-Pearson, las pruebas de hipótesis se planten como un proceso de decisión entre dos hipótesis, en él se consideran una hipótesis alternativa $(\mathrm{H} 1)$ que es la negación o complemento de la hipótesis nula $(\mathrm{Ho})$. Se definen regiones de rechazo y no rechazo sobre la distribución muestral del estadístico de prueba y los siguientes tipos de errores que se pueden cometer (Inzunsa y Jiménez, 2013). Para la presente investigación se formularon las siguientes hipótesis a comprobar:

H1. Más del 60\% de los colaboradores no se encuentran motivados en su entorno laboral.

Ho. Más del $60 \%$ de los colaboradores se encuentran motivados en su entorno laboral.

Tabla 3. Test and $\mathrm{Cl}$ for One Proportion de no estar motivados con su entorno laboral

Test of $p=0,6$ vs $p>0,6$

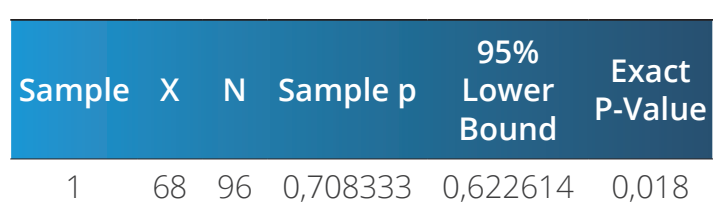

Fuente: Elaboración propia (2019)

Con los resultados obtenidos en la prueba, se rechaza la hipótesis nula porque el P-value (0.018) es menor que el nivel de significancia (0.05), por lo que se concluye que más del $60 \%$ 
de las personas encuestadas no están motivados con su entorno laboral, esto es un dato significativo y que debe ser tema de análisis ya que 6 de cada 10 colaboradores en las medianas empresas de Xalapa no encuentran una motivación en su ámbito laboral.

Los resultados de la prueba deben ser tomados en cuenta por los empleadores, ya que de acuerdo con Miranda (2016) en las empresas no se debe dar exclusivamente importancia a los factores que se creen fundamentales para su éxito como la maquinaria, los recursos financieros, el terreno, materias primas y más; la mayor importancia debe ser el recurso humano, puesto que las personas son lo más importante y constituyen el elemento primordial para el éxito empresarial.

\section{Discusión}

Sin duda es importante hacer mención que la presente investigación tiene sus limitaciones al no poder realizar un comparativo referente a lo que los empleadores tienen de percepción sobre si los colaboradores se encuentran motivados subiendo de nivel en la pirámide de satisfacción, por lo que se ve en la necesidad de realizar ese estudio que contraponga las percepciones de ambas partes para tener mayores elementos sobre el impacto que tiene la motivación en los colaboradores. Un aspecto de valor de esta investigación es sobre el diseño del instrumento al cual se le aplicaron las técnicas de validación por Alfa de Cronbach con resultados favorables lo que se interpreta que un ámbito de tipo social se puede medir con menor incertidumbre las dimensiones establecidas, en el mismo sentido al realizar la prueba de hipótesis, permitió comprobar qué tan motivado se siente los colaboradores resultado que debe ser considerado para proponer estrategias en los planes de desarrollo del recurso humano, de igual forma los propios resultados descriptivos de la investigación son una fortaleza para la planeación a corto plazo que deben hacer empleadores sobre cómo se debe ir motivando a sus colaboradores.

Referente a la motivación laboral, $74 \%$ de los colaboradores se encuentran muy satisfechos en su ámbito laboral y de acuerdo con la literatura coincidiendo con Marin, Helem y Plascencia (2017), se espera que tengan un mayor rendimiento y desempeño laboral.

\section{Conclusión}

Esta investigación, permitió constatar que las teorías propuestas por Maslow tienen significancia en los aspectos de motivación, ya que las empresas han buscado el punto en que las actividades de sus colaboradores sean equilibradas, ellos ganan y sus colaboradores también, crecen en conjunto, los motivan a que no se estanquen y estos se desarrollen bajo mejores ambientes laborales. Sin embargo podemos verificar que esta intención se da en un aspecto solo propositivo por parte de las empresas, ya que si se analizan los resultados de la investigación, se comprobó con la prueba de hipótesis estadística 
que más del $60 \%$ de las personas encuestadas no están motivados con su entorno laboral, en el mismo sentido y de acuerdo a la tendencia en la satisfacción de las necesidades, estas no muestran un equilibrio sistemáti$\mathrm{co}$, ya que como se pudo apreciar la tendencia es descendente conforme se va subiendo de nivel en la pirámide, por lo que las empresas deben tomar seriamente en cuenta que deben implementar cambios necesarios en la forma de operar para que sus trabajadores puedan encontrar su sentido de pertenencia, de superación y autorrealización, y no solo satisfacer sus necesidades básicas.

Para mejorar la motivación laboral de los colaboradores que no se encuentran motivados, y alienado a lo que Marin, Helem y Plascencia (2017) proponen se puede sugerir el enriquecimiento de trabajo que permitirá otorgar mayores responsabilidades al empleado, dando tareas cada vez más complejas de manera que este encuentre la oportunidad de satisfacer sus necesidades de crecimiento personal, todo ello con el diseño programas de desarrollo organizacional. Para esto, Herzberg incluso, señala 7 estrategias: eliminación de controles innecesarios, aumento de la responsabilidad, asignación de los empleados a unidades naturales y completas de trabajo (sección, división, etc), conceder más autonomía en las actividades, elaboración de informes periódicos sobre calidad de desempeño, introducción de nuevas e interesantes tareas y asignación a tareas especializadas.
Esta investigación queda abierta para llevar a cabo un proyecto de intervención en alguna de las empresas para que se implementen los mecanismos bajo propuestas que permitan en la medida de lo posible implementar estrategias con el fin de que los colaboradores puedan satisfacer con las necesidades no sólo básicas sino también aquellas de autorrealización que les permitan estar realmente motivados en su ámbito laboral.

\section{Referencias}

Aguilar Salmerón, Guadalupe de la Cruz (2018). Desarrollo Humano y Creatividad. Una aproximación humanística. El Artista, (15), undefined-undefined. [fecha de Consulta 6 de octubre de 2019]. ISSN: Disponible en: https://www.redalyc.org/articulo. oa?id=874/87457958002

Aguilera, V. (2011). Liderazgo y Clima Organizacional en las Instituciones Educativas de la Fundación Creando Futuros (Tesis doctoral, Universidad de Alcalá, España). Recuperada de file:///C:/Users/Tirso/Downloads/ Tesis\%2 Odoctoral\%20V\%C3\%ADctor\%20Aguilera.pdf

Ávila Martínez, K. (2015). Impacto del tratado de libre comercio -tlc- en la actividad logistica portuaria de colombia. Dictamen Libre, (17), 31-39. https://doi.org/10.18041/2619-4244/ dl.17.3078

Arauz Aismeth, Diaz Marissa, GonzalezMariel y Vasquez Yelipssa (2018). Evaluación y análisis de los indicadores de motivación laboral aplicado al personal operativo de restaurantes de comida rápida. Gente Clave. Revista Académica del Centro de Estudios de 
Postgrado. Universidad Latina de Panamá, 3 (1), 16-29

Blanco Rangel, I. (2016). Apuntes sobre Colombia a propósito de su internacionalización y otros desafíos. Aglala, 7(1), 209-224. Recuperado de http:// revistas.curnvirtual.edu.co/index.php/ aglala/article/view/905

Boeree, G. (2006). Personality Theories Psychology Department Shippensburg University. Documento en línea recuperado 12 noviembre de 2019 de http://www.ship.edu/\%7Ecgboeree/ perscontents.html

Celina H. y Campo A., (2005), "Aproximación al uso del coeficiente alfa de Cronbach", Revista colombiana de psiquiatría, vol. XXXIV, número 004, Asociación Colombiana de Psiquiatría, Bogotá, Colombia, pp. 572 - 580, disponible en: http://redalyc.uaemex.mx/ pdf/806/80634409.pdf

Daft, L. Administración. Sexta edición. Thompson, 2004

Feist, J. \& Feist, G. (2006) Theories of Personality (sexta edición). New York: McGraw Hill.

Ferrell, O. C. y Hirt, G. (2003) Introducción a los negocios en un mundo cambiante. Cuarta edición. McGraw-Hill, 2003.

García Cadena, Cirilo H. (2006). La medición en ciencias sociales y en la psicología. Estadística con SPSS y metodología de la investigación. México. Trillas

Gómez Nieto, Begoña, \& Benito Vielba, Cristina (2014). Presente de la comunicación organizacional en la pyme española. Razón y Palabra, (86), undefined-undefined. [fecha de Consulta 26 de septiembre de 2019]. ISSN: 1605-4806. Disponible en: http://www.redalyc.org/articulo. orcid=1995/199530728023

Inzunsa Cazares, S., \& Vidal Jiménez Ramírez, J. (2013). Caracterización del razonamiento estadístico de estudiantes universitarios acerca de las pruebas de hipótesis. Revista Latinoamericana de Investigación en Matemática Educativa, 16 (2), 179-211. https://dx.doi. org/10.12802/relime.13.1622

José Rafael Quintero Angarita. (2015). TEORÍA DE LAS NECESIDADES DE MASLOW. TEORÍA DE LAS NECESIDADES DE MASLOW, III, pp.1-6. Disponible: http://files.franklinyagua.webnode.com.ve/200000092e266ae35e3/ Teoria_Maslow_Jose_Quintero.pdf

Logroño Varela, Elena, \& Cercas Duque, Adriana. (2018). Estudio de la motivación del cuidador principal de la persona dependiente. Ene, 12(1), 787. Epub 23 de abril de 2018. Recuperado en 21 de mayo de 2020, de http://scielo.isciii.es/scielo.php?script=sci_arttext\&pi$d=S 1988-348 \times 2018000100008 \& \mid n-$ $\mathrm{g}=\mathrm{es} \& \operatorname{ting}=e s$.

Marin Samanez, Helen Stephani, \& Placencia Medina, Maritza Dorila. (2017). Motivación y satisfacción laboral del personal de una organización de salud del sector privado. Horizonte Médico (Lima), 17(4), 42-52. https:// dx.doi.org/https://doi.org/10.24265/ horizmed.2017.v17n4.08

Martínez Muñoz, E., Hernández Gracia, T., Torres-Flórez, D., García Velázquez, M. del R., \& Montaño Arango, O. (2018). La realidad comunicacional, liderazgo y clima laboral de la pequeña empresa industrial textil en el Estado de Hidalgo. Revista GEON (Gestión, Organizaciones Y Negocios), 5(1), 76-90. https:// doi.org/10.22579/23463910.15 
Maslow, A. H. "A theory of human motivation". Psychological Review 50. 1943: 370-396

Pineda Henao, A. (2020). La comunicación organizacional en la gestión empresarial: retos y oportunidades en el escenario digital. Revista GEON (Gestión, Organizaciones Y Negocios), 7(1), 9-25. https:// doi.org/10.22579/23463910.182
Polo Rodríguez, F., \& Villasmil Molero, M. (2018). Estudio comparado sobre la regulación del impuesto al valor agregado (IVA) en Colombia y España. Dictamen Libre, 2(23), 15-24. https://doi. org/10.18041/2619-4244/dl.23.5142

Robbins, S. P. y Coulter, M. Administración. (2005) Octava edición. Pearson Prentice Hall, 2005. 